\title{
Convexidade generalizada e condições de otimalidade para problemas multiobjetivos
}

\author{
Camila Isoton, Lucelina Batista Santos, \\ Depto de Matemática, UFPR, \\ 81531-980, Curitiba, PR \\ E-mail: isoton.camila@gmail.com, lucelina@ufpr.br.
}

Resumo: Apresentamos um estudo sobre o papel das funções convexas generalizadas no estudo de problemas multiobjetivos. Inicialmente, caracterizaremos a eficiência fraca em termos de escalarização. É sabido que, sob hipótese de convexidade, toda solução fracamente eficiente é solução do problema ponderado. Mais geralmente, um problema multiobjetivo é KT-invexo se e somente se toda solução fracamente eficiente é solução de um problema ponderado. Consideramos também as condições necessárias de otimalidade de 1a. e de 2a. ordem. É bastante conhecido que, sob hipóteses de convexidade, as condições de Kuhn-Tucker são suficientes para a otimalidade. Consideraremos a classe dos problemas KT-pseudoinvexos, os quais possuem a seguinte propriedade: um problema é KT-invexo se, e somente se, todo ponto Kuhn-Tucker é solução fracamente eficiente do problema multiobjetivo. Além disto, vale um resultado semelhante a este, utilizando-se as condições necessárias de 2a. ordem. Veremos que um problema multiobjetivo é KT-pseudoinvexo de segunda ordem se, e somente se, todo ponto Kuhn-Tucker de segunda ordem é solução fracamente eficiente. Na obtenção destes resultados, os teoremas de alternativa foram amplamente utilizados.

Palavras-chave: Condições de Otimalidade; otimização mono e multiobjetivos; funções convexas generalizadas, KT-invexidade.

\section{INTRODUÇÃO}

Diariamente encontramos situações que conduzem à tomada de decisões. Em inúmeras situações, o decisor fixa vários objetivos, os quais são estabelecidos como metas a serem atingidas. Assim, é grande a aplicabilidade de problemas multiobjetivos (também chamados problemas vetoriais ou problemas com objetivos múltiplos) e daí o nosso interesse em estudá-los.

Formalmente, tais problemas admitem a seguinte formulação:

$$
\begin{aligned}
& \text { Minimizar } f(x):=\left(f_{1}(x), \cdots, f_{p}(x)\right) \\
& \text { sujeito a: } x \in S
\end{aligned}
$$

onde $f_{j}: \mathbb{R}^{n} \rightarrow \mathbb{R}$ e $S$ é um subconjunto não vazio de $\mathbb{R}^{n}$.

Em geral, não é possível minimizar simultaneamente todos os objetivos. Deste modo, existem diferentes noções de otimalidade (eficiência) associadas ao problema (MOP). Consideraremos neste trabalho os conceitos de solução eficiente, fracamente eficiente e propriamente eficiente. Além disto, admitiremos que o conjunto factível $S$ é descrito por restrições de desigualdade,

$$
S=\left\{x \in \mathbb{R}^{n}: g_{i}(x) \leq 0, i=1, \cdots, m\right\}
$$

e $g_{i}: \mathbb{R}^{n} \rightarrow \mathbb{R}$ são funções dadas. Para tais problemas, condições de otimalidade são dadas na forma de regra de multiplicadores - são as chamadas condições de Kuhn-Tucker, para o caso onde 
as funções são de classe $C^{1}$ Para o caso $C^{2}$, também existe um resultado análogo, utilizando as informações das derivadas de segunda ordem das funções. Veja Chankong and Haimes [1] e Wang [8], por exemplo.

É bastante conhecida a importância das funções convexas na Teoria da Otimização. Para problemas convexos, a otimalidade local implica a otimalidade global. Além disto, as condições necessárias de otimalidade (por exemplo, as condições de Kuhn-Tucker) são também suficientes. Neste contexto, as funções invexas introduzidas por Hanson (1981) [2] são particularmente interessantes. Com efeito, Hanson demonstrou que uma função é invexa se e somente se todo ponto estacionário da função é ponto de mínimo global. Além disto, para problemas invexos com restrições de desigualdade, as condições de Kuhn-Tucker são suficientes para a otimalidade global. Entretanto, tais condições não são necessárias para a otimalidade, nem mesmo quando todas as funções do problema são invexas. Martin (1985) [5] propõe uma ligeira modicação do conceito de invexidade e introduz a noção de problemas KT-invexos e demonstra que um problema é KT-invexo se, e somente se, todo ponto Kuhn-Tucker é minimizador global.

Resultados similares a estes foram estabecidos com as condições de otimalidade de segunda ordem. Ivanov (2011) [4] introduz o conceito de problemas KT-invexos de segunda ordem e demonstra que o problema é KT-invexo de segunda ordem se e somente se todo ponto KuhnTucker de segunda ordem é minimizador global.

O objetivo deste trabalho é apresentar generalizações dos resultados obtidos por Martin [5] e Ivanov [4] para o problema multiobjetivo (MOP).

\section{RESULTADOS}

\subsection{PRELIMINARES TÉCNICOS}

Abaixo são definidas algumas noções de otimalidade:

- eficiência fraca: $x^{*} \in S$ é chamada solução fracamente eficiente de (MOP) se não existe $x \in S$ tal que $f_{j}(x)<f_{j}\left(x^{*}\right), j=1, \cdots, p$.

- eficiência: $x^{*} \in S$ é solução eficiente de (MOP) se não existe $x \in S$ tal que $f_{j}(x) \leq$ $f_{j}\left(x^{*}\right), j=1, \cdots, p$ com desigualdade estrita válida para algum índice $j$.

- eficência própria: $x^{*} \in S$ é solução propriamente eficiente de (MOP) se $x^{*}$ é eficiente e se existe um escalar $M>0$ tal que para cada $i$,

$$
\frac{f_{i}\left(x^{*}\right)-f_{i}(x)}{f_{j}(x)-f_{j}\left(x^{*}\right)} \leq M
$$

para algum $j$ tal que $f_{j}(x)>f_{j}\left(x^{*}\right)$, sempre que $x$ é factível para (MOP) e $f_{i}(x)<f_{i}\left(x^{*}\right)$.

É evidente que eficiência própria implica eficiência e eficiência implica eficiência fraca. As recíprocas são falsas em geral. Neste trabalho, são consideradas as noções de eficiência fraca.

Uma possível abordagem para a determinação das soluções de (MOP) é a escalarização. Por escalarização, entende-se um processo através do qual é possível converter um problema multiobjetivo em um problema escalar equivalente, de modo que as soluções de (MOP) podem ser obtidas como soluções de problemas clássicos de programação matemática. Talvez, a mais conhecida destas técnicas seja a ponderação. Para cada $\omega \in \mathbb{R}_{+}^{P} \backslash\{0\}$, definiremos:

$$
\begin{aligned}
& \text { Minimizar } \sum_{i=1}^{p} \omega_{i} f_{i}(x) \\
& \text { sujeito a: } x \in S .
\end{aligned}
$$

Adotaremos a seguinte notação: Para $x, y \in \mathbb{R}^{n}$, escreveremos

$$
\begin{aligned}
& x<y \Longleftrightarrow x_{i}<y_{i}, i=1, \cdots, n \\
& x \leqq y \Longleftrightarrow x_{i} \leq y_{i}, i=1, \cdots, n \\
& x \leq y \Longleftrightarrow x \leqq y \text { e } x \neq y .
\end{aligned}
$$


É bastante conhecido o seguinte resultado:

Lema 1. (i) Se existe um vetor $\omega \geqq 0, \omega \neq 0$ tal que $x^{*} \in S$ é solução de $\left(P_{\omega}\right)$, então $x^{*} e ́$ solução fracamente eficiente de (MOP).

(ii) Se as funções $f_{j}, j=1, \cdots, p$ são convexas e $x^{*} \in S$ com $S$ convexo, solução fracamente eficiente de (MOP), então existe um vetor $\omega \geqq 0, \omega \neq 0$ tal que $x^{*}$ é solução do problema ponderado $\left(P_{\omega}\right)$.

Para detalhes, veja Chankong e Haimes [1].

Lema 2. (i) [Condições de 1a. ordem] Suponha que o problema (MOP) é de classe $C^{1}$. Se $x^{*} \in S$ é uma solução fracamente eficiente de (MOP), então existem multiplicadores $\lambda \in \mathbb{R}_{+}^{p}, \mu \in \mathbb{R}_{+}^{m}$, não simultaneamente nulos e tais que

$$
\begin{gathered}
\sum_{j=1}^{p} \lambda_{j} \nabla f_{j}\left(x^{*}\right)+\sum_{i=1}^{m} \mu_{i} \nabla g_{i}\left(x^{*}\right)=0, \\
\mu_{i} g_{i}\left(x^{*}\right)=0, i=1, \cdots, m .
\end{gathered}
$$

(ii) [Condições de 2a. ordem] Suponha que (MOP) é de classe $C^{2}$. Seja $x^{*} \in S$ uma solução fracamente eficiente de (MOP). Então para cada $d \in D\left(x^{*}\right)$, existem vetores $\lambda \geqq 0, \mu \geqq 0$, não todos nulos e tais que:

$$
\begin{gathered}
\sum_{j=1}^{p} \lambda_{j} \nabla f_{j}\left(x^{*}\right)+\sum_{i=1}^{m} \mu_{i} \nabla g_{i}\left(x^{*}\right)=0 \\
\mu_{i} g_{i}\left(x^{*}\right)=0, i=1, \cdots, m \\
\mu_{j} \nabla^{T} f_{j}\left(x^{*}\right) d=0, j=1, \cdots, p \\
\lambda_{i} \nabla^{T} g_{i}\left(x^{*}\right) d=0, i \in 1, \cdots, m \\
d^{T}\left[\sum_{j=1}^{p} \lambda_{j} \nabla^{2} f_{j}\left(x^{*}\right)+\sum_{i=1}^{m} \mu_{i} \nabla^{2} g_{i}\left(x^{*}\right)\right] d \geq 0 .
\end{gathered}
$$

(onde $I\left(x^{*}\right)=\left\{i: g_{i}\left(x^{*}\right)=0\right\}$ é o conjunto das restrições ativas em $x^{*}$ e $D\left(x^{*}\right)=\left\{d \in \mathbb{R}^{n}\right.$ : $\left.\nabla^{T} f_{j}\left(x^{*}\right) d \leq 0, j=1, \cdots, p, \nabla^{T} g_{i}\left(x^{*}\right) d \leq 0, i \in I\left(x^{*}\right)\right\}$ é o conjunto das direções críticas em $\left.x^{*}\right)$.

Para detalhes: Veja Chankong e Haimes [1]e Wang [8].

Definição 1. (i) Um ponto $x^{*} \in S$ satisfaz (1),(2), com $\lambda \neq 0$ é chamado ponto Kuhn-Tucker de $(M O P)$.

(ii) Se $x^{*} \in S$ satisfaz (3)-(7) com $\lambda \neq 0$, para cada direção crítica $d \in D\left(x^{*}\right)$, então $x^{*}$ é chamado ponto Kuhn-Tucker de segunda ordem de (MOP).

Neste trabalho, estudamos as relações existentes entre convexidade generalizada e os problemas ponderados. Também discutiremos as relações existentes entre os conceitos de pontos Kuhn-Tucker (de primeira e de segunda ordem) e a convexidade generalizada.

\subsection{ESCALARIZAÇÃO}

Osuna-Gómez et al. (1999) introduzem em [6] o conceito de problemas multiobjetivos KTinvexos:

Definição 2. O problema (MOP) é KT-invexo, se para quaisquer $x, y \in S$, existe um vetor $\eta(x, y) \in \mathbb{R}^{n}$ tal que

$$
\begin{gathered}
f_{j}(y)-f_{j}(x) \geq \nabla^{T} f_{j}(x) \eta(y, x), j=1, \cdots, p \\
-\nabla^{T} g_{i}(x) \eta(y, x) \geq 0, i \in I(x) .
\end{gathered}
$$


Observe que esta é a extensão natural do conceito de problemas (escalares) KT-invexos introduzido por Martin [5].

Teorema 1. (MOP) é KT-invexo se e somente se toda solução fracamente eficiente de (MOP) é solução de um problema ponderado $\left(P_{\omega}\right)$, para algum $\omega \geqq 0, \omega \neq 0$.

Esboço da Prova.

$(\Rightarrow)$

- Seja (MOP) KT-invexo, então $\bar{x} \in X$ ponto Kuhn-Tucker resolve um problema escalar ponderado. (A prova segue da definição de ponto Kuhn-Tucker).

$(\Leftarrow)$

- Suponha que $\bar{x} \in X$ ponto Kuhn-Tucker é solução fracamente eficiente e resolve $\left(P_{w}\right)$ para algum $w \in W$. Então o seguinte sistema não tem solução $(\lambda, v) \geq 0, v>0$

$$
\left\{\begin{array}{l}
\sum_{j \in J} \lambda_{j} \nabla f_{j}(\bar{x})+\sum_{i \in I(\bar{x})} \mu_{i} \nabla g_{i}(\bar{x})=0 \\
v+\sum_{j \in J} w_{j}\left(f_{j}(x)-f_{j}(\bar{x})\right)=0
\end{array}\right.
$$

$\forall \bar{x}, x \in X$ e $\forall j \in J=\{j: j=1, \ldots, p\}$.

- E aplicando o Teorema de Motzkin segue que o sistema abaixo tem solução $(\eta, \varepsilon), \eta \in \mathbb{R}^{n}$ e $\varepsilon \in \mathbb{R}$

$$
\left\{\begin{array}{l}
\varepsilon<0 \\
\nabla^{T} f_{j}(\bar{x}) \eta+\varepsilon\left(f_{j}(x)-f_{j}(\bar{x})\right)<0, \forall j \in J \\
\nabla^{T} g_{i}(\bar{x}) \eta \leqq 0, i \in I(\bar{x})
\end{array}\right.
$$

$\forall \bar{x}, x \in X$

- e com isto, chegamos a conclusão de que (MOP) é KT-invexo.

\subsection{CONDIÇÕES DE 1A. ORDEM}

Osuna-Gómez et al. introduzem em [6] o conceito de problemas KT-pseudoinvexos:

Definição 3. O problema (MOP) é KT-pseudoinvexo se, para quaisquer $x, y \in S$ existe um vetor $\eta(x, y) \in \mathbb{R}^{n}$ tal que :

$$
\begin{gathered}
f_{j}(y)<f_{j}(x) \Longrightarrow \nabla^{T} f_{j}(x) \eta(x, y)<0, j=1, \cdots, p \\
-\nabla^{T} g_{i}(x) \eta(x, y) \geq 0, i \in I(x) .
\end{gathered}
$$

Esta é a mais ampla classe de problemas para os quais as condições de Kuhn-Tucker são necessárias e suficientes para a eficiência fraca:

Teorema 2. (MOP) é KT-pseudoinvexo se, e somente se, todo ponto Kuhn-Tucker é solução fracamente eficiente de (MOP).

Esboço da Prova.

$(\Leftarrow)$

- Assumimos que dados $x, \bar{x} \in X$, com $f_{j}(x)<f_{j}(\bar{x}), \forall j \in J$, temos que $\bar{x}$ não é solução fracamente eficiente de (MOP) e, por hipótese, $\bar{x}$ não é ponto Kuhn-Tucker, e assim, não valem as condições de KKT. 
- Então, podemos aplicar o Teorema de Motzkin para garantir a solubilidade do sistema

$$
\left\{\begin{array}{c}
\nabla^{T} f_{j}(\bar{x}) d<0, j \in J \\
\nabla^{T} g_{i}(\bar{x}) d \leq 0, i \in I(\bar{x})
\end{array}\right.
$$

- E basta definir $\eta(x, \bar{x})=d$, que obtemos o resultado desejado.

$(\Rightarrow)$

- Suponhamos que o problema (MOP) é KT-pseudoinvexo e que $\bar{x} \in X$ é um ponto KuhnTucker de (MOP). Então concluímos que $\bar{x}$ é solução fracamente eficiente de (MOP).(A prova deste fato se dá por absurdo, supondo que $\bar{x} \in X$ é ponto de Kuhn-Tucker porém não é solução fracamente eficiente)

\subsection{CONDIÇÕES DE 2A. ORDEM}

Numa abordagem bastante semelhante às utilizada por Osuna-Gómez et al. [6] e Ivanov [4], Santos et al. [7] propõem o conceito de problemas KT-pseudoinvexos de segunda ordem.

Definição 4. Dizemos que o problema (MOP) é KT-pseudoinvexo de segunda ordem se para quaisquer $x, y \in S$, com $f_{j}(y)<f_{j}(x), j=1, \cdots, p$ existem uma direção $d(x, y) \in D(x)$, um número não negativo $\omega(x, y)$ e um vetor $\eta(x, y) \in \mathbb{R}^{n}$ tais que

$$
\begin{gathered}
\nabla^{T} f_{j}(x) \eta(x, y)+\omega(x, y) d^{T}(x, y) \nabla^{2} f_{j}(x) d(x, y)<0, j=1, \cdots, p \\
\nabla^{T} g_{i}(x) \eta(x, y)+\omega(x, y) d^{T}(x, y) \nabla^{2} g_{i}(x) d(x, y) \leq 0, i \in I(x) .
\end{gathered}
$$

Esta é a mais ampla classe de funções para as quais as condições de Kuhn-Tucker de segunda ordem são necessárias e suficientes para a eficiência fraca:

Teorema 3. (MOP) é KT-pseudoinvexo de segunda ordem se e somente se todo ponto KuhnTucker de segunda ordem é solução fracamente eficiente de (MOP).

Esboço da Prova.

$(\Rightarrow)$

- Suponha por contradição que $\bar{x} \in X$ é um ponto Kuhn-Tucker de segunda ordem, mas não é solução fracamente eficiente. Então, existe $y \in X$ tal que $f(y)<f(\bar{x})$, e da KTpesudoinvexidade de segunda ordem, existem $d(\bar{x}, y) \in D(\bar{x}), \omega(\bar{x}, y) \in \mathbb{R}_{+}$e $\eta(\bar{x}, y) \in \mathbb{R}^{n}$ tais que

$$
\begin{gathered}
\nabla f_{j}(\bar{x}) \eta(\bar{x}, y)+\omega(\bar{x}, y) d^{T}(\bar{x}, y) \nabla^{2} f_{j}(\bar{x}) d(\bar{x}, y)<0, j \in J \\
\nabla g_{i}(\bar{x}) \eta(\bar{x}, y)+\omega(\bar{x}, y) d^{T}(\bar{x}, y) \nabla^{2} g_{i}(\bar{x}) d(\bar{x}, y) \leq 0, \quad i \in I(\bar{x}) .
\end{gathered}
$$

- Seja $(\lambda, \mu)$, o par de multiplicadores associados à direção crítica $d(\bar{x}, y)$. Se multiplicamos (10) por $\lambda_{j} \geq 0$ e (11) por $\mu_{i} \geq 0$ e, somando as desigualdades obtidas e rearranjando os termos chegamos no absurdo.

$(\Leftarrow)$

- Fixamos $\bar{x} \in X$ e $d \in D(\bar{x})$. e consideramos os seguintes sistemas:

$$
\left.\begin{array}{c}
\nabla^{T} f_{j}(\bar{x}) \eta+\omega d^{T} \nabla^{2} f_{j}(\bar{x}) d<0, j \in J \\
\nabla^{T} g_{i}(\bar{x}) \eta+\omega d^{T} \nabla^{2} g_{i}(\bar{x}) d \leq 0, i \in I(\bar{x})
\end{array}\right\}
$$

em que $\eta \in \mathbb{R}^{n}$ e $\omega \in \mathbb{R}_{+}$e o sistema

$$
\left.\begin{array}{c}
f_{j}(y)-f_{j}(\bar{x})<0, j \in J \\
g_{i}(y) \leq 0, i \in I(\bar{x})
\end{array}\right\}
$$

em que $y \in U$. 
- Pelo Teorema de Motzkin não homogêneo $\bar{x} \in X$ é um ponto Kuhn-Tucker de segunda ordem $\Leftrightarrow\left(\mathrm{S}_{1}\right)$ não tem solução $(\eta, \omega)$.

- Por outro lado, $\bar{x} \in X$ é solução fracamente eficiente de $(\mathrm{MOP}) \Leftrightarrow$ o sistema $\left(\mathrm{S}_{2}\right)$ não tem solução $y$.

- Como por hipótese, todo ponto Kuhn-Tucker de segunda ordem é solução fracamente eficiente de (MOP), os sistemas $\left(\mathrm{S}_{1}\right)$ e $\left(\mathrm{S}_{2}\right)$ são equivalentes. Portanto, (MOP) é KTpseudoinvexo de segunda ordem.

\section{CONCLUSÕES}

Neste trabalho, discutimos as relações existentes entre a convexidade generalizada e as soluções de problemas multiobjetivos. Verificamos que a convexidade generalizada desempenha um importante papel na caracterização da eficiência em termos de problemas escalarizados.

Também estudamos condições necessárias de primeira e de segunda ordem para problemas multiobjetivos e caracterizamos as mais amplas classes de problemas multiobjetivos para as quais as condições de Kuhn-Tucker (de primeira e de segunda ordem) resultam suficientes para a otimalidade.

Como possibilidade de trabalho futuro, pretendemos adequar estas técnicas a outras classes de problemas de otimização, como por exemplo, o problema de otimização vetorial (entre espaços abstratos), o problema de programação infinita e o problema de otimização em tempo contínuo.

Nas demonstrações dos principais resultados apresentados neste trabalho (e discutidos com maiores detalhes na Dissertação [3]) observamos que são necessários, basicamente, resultados de condições necessárias de otimalidade (de primeira e de segunda ordem) e Teoremas de Alternativas. Existem variantes destes resultados para os problemas citados anteriormente, donde cremos, ser possível estender os resultados estudados para tais problemas. Inclusive, cremos ser possível estabelecer tais resultados, com hipóteses relaxadas de diferenciabilidade.

\section{Referências}

[1] V. Chankong, Y. Y. Haimes. Multiobjective decision-making: theory and methodology. North-Holland, Amsterdam, 1983.

[2] M. A. Hanson. On sufficiency of Kuhn-Tucker conditions, J.Math. Anal. Appl. 80, 1981, p. $545-550$.

[3] C. Isoton. Condições necessárias e suficientes de otimalidade para problemas com um e com vários objetivos. Dissertação de Mestrado. Universidade Federal do Paraná, 2013.

[4] V. I. Ivanov. Second-order Kuhn-Tucker invex constrained problems. J. Global Optim. 50, 2011, p. 519-529.

[5] D. H. Martin. The essence of invexity. Journal of Optimization Theory and Applications. Vol. 47, no. 1, 1985, p. 65-76.

[6] R. Osuna-Gómez, A. Beato-Moreno, A. Rufián-Lizana. Generalized convexity in multiobjective programming. J. Math. Anal. Appl. 233, 1999, p. 205-220.

[7] L. B. Santos, R. Osuna-Gómez, B. Hernández-Jiménez e M. Rojas-Medar. Necessary and sufficient second-order optimality conditions for multiobjective problems with $C^{1}$ data. Nonlinear Analysis 85, 2013, p. 192-203.

[8] S. Wang. Second-order necessary and sufficient conditions in multiobjective programming. Numer. Func. Anal. and Optimiz. 12, 1991, p. 237-252. 